\title{
GENERATING REUSE CONCEPTS FOR RETIRED WIND-TURBINE BLADES
}

\author{
S.R.X. Jiang \\ Mechanical \& Industrial Engineering \\ University of Toronto \\ Toronto, Canada
}

\author{
David Inkermann \& Thomas Vietor \\ Institute for Engineering Design \\ Technische Universität Braunschweig \\ Braunschweig, Germany
}

\author{
L.H. Shu* \\ Mechanical \& Industrial Engineering \\ University of Toronto \\ Toronto, Canada \\ *shu@mie.utoronto.ca
}

\begin{abstract}
This work examines identifying reuses for wind-turbine blades that are retired when they reach the end of their technical life. Wind-turbine blades are made from fibercomposite materials, for which effective material-based recycling technologies are extremely limited. An approach to avoid or postpone recycling is to repurpose wind-turbine blades in other applications, as they should not be reused as windturbine blades for safety reasons.
\end{abstract}

However, identifying promising reuses for wind-turbine blades is challenging due to their specific shape properties. In addition to issues of functional fixedness, wind-turbine blades are physically much larger than everyday objects with which people typically reason. Following a series of studies where engineering students were asked to identify wind-turbine-blade reuses, a method involving perspective-taking was developed and applied. The effects of this method on concept generation are reported and compared to SCAMPER, an existing design method. To better understand and motivate human designers, this work also incorporates psychological concepts, including Need for Closure and Regulatory Focus Theory.

Keywords: Sustainability, Component reuse, Wind-turbine blades, Concept generation, Functional fixedness

\section{INTRODUCTION}

Global installation of wind-turbine technology is expected to continue to increase, decreasing reliance on energy sources that contribute to climate change. However, wind-turbine blades of up to 100 meters in length are typically manufactured using glass-fiber reinforced polymer (GFRP). While GFRP provides structural integrity, it is also comprised of thermoset resins, which is difficult to reform or recycle. Although wind turbines are maintained over their use life for effective operation, they are eventually retired based on durability and prediction models. Ramirez-Tejeda et al. (2017) estimates that by 2028, retired wind-turbine blades will comprise 330,000 tons/year of composite waste. Recycling of retired wind-turbine blades for their scrap material is challenging since GFRP can only be reformed through machining, which destroys reinforcing fibers and compromises the material's integrity.

Funded by Natural Sciences and Engineering Research Council of Canada (NSERC)
For the above reasons, the current work explores the process of identifying possible repurposes or reuses for retired windturbine blades. In contrast to other cases of reuse, wind-turbine blades should not be reused in the same application, i.e., as windturbine blades nor aircraft components for safety reasons. While retired wind turbines have been repurposed as children's play structures and benches for public seating, such reuses alone will not likely fully absorb the supply of retired wind-turbine blades.

\section{BACKGROUND}

This work combines design-related and social-psychology concepts to better understand and motivate designers. Thus, we begin with background on the seemingly disparate concepts before describing how they are incorporated in the current work.

\section{A. Functional Fixedness}

Adamson (1952) described functional fixedness as a tendency to focus on an object's previously known function, which inhibits discovering appropriate new uses for this object. Related to design fixation, functional fixedness often leads designers to repeat the same set of solutions they identified, unable to see more creative solutions. Specific to the reuse of wind-turbine blades, individuals who are strongly affected by functional fixedness may have trouble identifying reuses other than as wind-turbine blades.

\section{B. SCAMPER}

SCAMPER is an acronym that corresponds to: Substitute, Combine, Adapt, Modify, Put to other use, Eliminate, and Reverse. SCAMPER is often applied to make modifications in configuration design, but it also has potential to help identify reuses for products. That is, each word or phrase of the acronym can be applied to a product or part to trigger potential reuses. Moreno et al. (2016) observed SCAMPER's effectiveness in generating novel concepts, since reflecting on each corresponding word or phrase could provide novel insights.

\section{Regulatory Focus Theory (RFT)}

A motivational framework developed by social psychologist Higgins (2000), Regulatory Focus Theory (RFT) has two independent self-regulatory orientations: prevention and promotion. This framework adds a dimension to the hedonistic model of approaching pleasure and avoiding pain. In RFT, a prevention-focused individual would approach safety and security (non-losses) and avoid losses. In contrast, a promotion- 
focused individual would approach hopes and accomplishments (gains) and avoid stagnation (non-gains).

In a variety of applications over the past two decades, Higgins et al. have demonstrated that increased task engagement results when individuals' regulatory focus and task orientation align. Thus, appropriate framing of a design task may improve the engagement of designers with different regulatory foci.

\section{Need for Closure (NFC)}

Individuals in general have different needs when it comes to obtaining firm answers to questions. Webster and Kruglanski (1994) developed a scale, called Need for Closure (NFC) that aims to measure these needs for cognitive closure and aversion to ambiguity. This scale has the following five subscales and corresponding sample statements: (1) order and structure: I enjoy having a clear and structured mode of life; (2) ambiguity: I'd rather know bad news than stay in a state of uncertainty; (3) decisiveness: I usually make important decisions quickly and confidently; (4) predictability: I don't like to go into a situation without knowing what I can expect from it; (5) closed mindedness: I do not usually consult many different options before forming my own view. To such statements, respondents select from choices that range from strongly disagree to strongly agree, with each choice contributing to their NFC score.

\section{E. Authors' Related Past Work}

Lai and Shu (2016) found that individuals with high NFC scores are more likely to fixate on a provided example when asked to develop a new concept. Olteteanu and Shu (2018) also linked subscales of the NFC to functional fixedness.

Olteteanu and Shu (2018) presented objects in various orientations, i.e., turned 0,90 and 180 degrees from a default orientation, and asked participants to identify alternative uses for the shown objects. Analysis of participant responses revealed that participants who were more receptive to reorientation cues were more likely to identify more, and more varied, uses for the objects. Thus, reorientation was shown to be an effective strategy to overcome functional fixedness in identifying alternative uses for everyday objects. These findings are consistent with those of Kudrowitz and Dippo (2013), whose Alternative-Uses-Test participants with more responses had more original responses.

Relating to the current study, wind-turbine blades are much larger than the everyday objects used in past AUT studies. In addition, people do not tend to interact with wind-turbine blades as they would with common everyday objects. Wind-turbine blades also rotate, i.e., change orientation during use, which may further reduce the effectiveness of using reorientation in the same way to decrease functional fixedness.

\section{METHODS}

A series of studies were conducted to gain insight into the process of generating reuse concepts for wind-turbine blades. This paper focuses on the fourth and fifth iterations, but major changes between iterations are summarized below to clarify previously encountered obstacles and how they were addressed. These studies serve to demonstrate the challenge in developing reuse concepts for a sustainable-energy object, which currently has few sustainable end-of-life options.

\section{A. Overview of Studies}

In each iteration of the study, engineering students were asked to identify reuses for parts of wind-turbine blades. They were shown drawings of four wind-turbine blade sections in at least one isometric and three orthogonal views. For parts with hollow sections, an additional isometric view was provided to show the section cut in half. The wind-turbine blades were presented as sections, since they are often cut into smaller pieces after being decommissioned for easier transportation. To convey part size, the first three iterations of the study used male and female CAD models as human scales. Since no difference in concepts were observed as a result of the different gendered models, the scale was replaced with a neutral stick figure to facilitate the introduction of other interventions. Fig. 1 shows the isometric views of all four sections, using this neutral figure to convey scale. Fig. 2 shows how the first part was presented to participants.

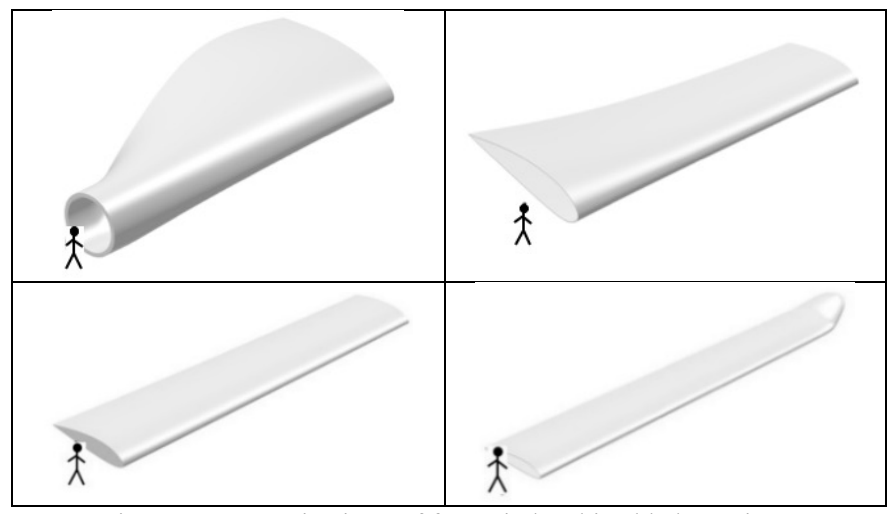

Figure 1. Isometric views of four wind-turbine blade sections.

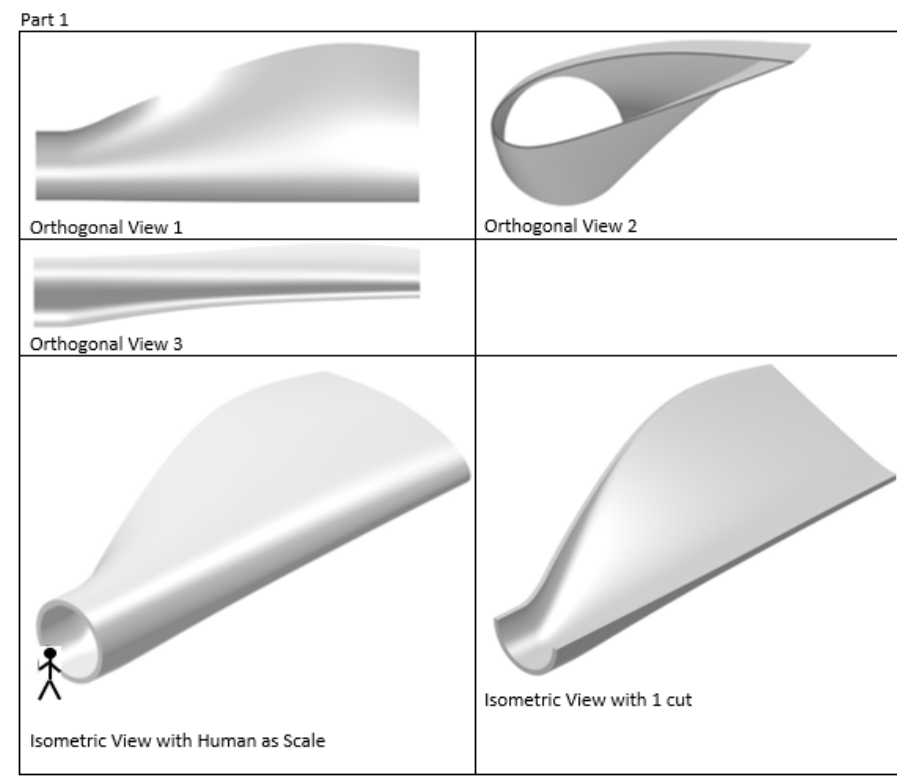

Figure 2. Different views of wind-turbine-blade Part 1.

\section{B. Previous Iterations of Study}

The first iteration of the study was conducted at Oldenburg University in Germany, in a graduate-level engineering course. To reduce functional fixedness, participants were not told that the parts were wind-turbine blades. For the same reason, they 
were not specifically prohibited from identifying reuses as windturbine blades. However, many engineering-student participants recognized the air-foil cross-section (in parts 2 and 3), and thus identified reuses as wind-turbine or airplane parts. Since the parts could not be reused in wind turbines, much less airplanes for safety reasons, these reuses were not feasible.

Thus, the second iteration, conducted in an engineering course at Bremen University in Germany, revealed that the parts were wind-turbine blades, and prohibited their reuse as the same or as airplane components. Although this restriction succeeded in reducing these specific reuse applications, the resulting reuse concepts either already exist, e.g., as children's play structures, or were impractical, e.g., as giant industrial mixing paddles.

Personal communication with a life-cycle engineering expert (Hauschild, 2017) towards more promising reuses led to the additional constraint that concepts address climate change. As this constraint could be expressed in multiple ways, they were formulated in line with Higgins' promotion versus prevention foci. Thus, third-iteration participants were asked to develop concepts that either "Enable pro-environmental behaviors to decrease the rate of climate change" (promotion focused), or "Help people cope with the inevitable results of climate change" (prevention focused). While this additional constraint provided direction and focus for concept generation, third-iteration participants in a 4th year undergraduate design course at the University of Toronto were observed to struggle when developing concepts. Thus, subsequent iterations explored existing (SCAMPER) and new (perspective-taking) methods to support this concept-generation activity.

\section{ITERATION FOUR USING SCAMPER}

The fourth iteration of the study was conducted at the Technische Universität Braunschweig in Germany.

\section{A. Methods}

Sixty-five (65) participants were recruited from a course on the basics of engineering design and product development, in a bachelor degree program. Of the 65,29 were asked to use SCAMPER, and the remaining 36 comprised the control group. However, the participants were known to be unfamiliar with SCAMPER. Therefore, those who were asked to use SCAMPER were given a four-page instructional aid, demonstrating how it could be applied towards concepts that reuse plastic bottles. Viswanathan et al. (2016) showed that examples with familiar features caused more fixation. Thus, examples in plastic-bottle reuse were chosen as they were dissimilar to wind-turbine blades in both function and scale. Fig. 3 shows examples for SCAMPER's "adapt" (similar features). In the top example, the cylindrical portion of the bottle is adapted for use as cylindrical magazine holders. In the bottom example, threads on the mouth of the bottle are adapted to fit with threads on a garden hose.

As was the case for the third iteration, participants were asked to complete the Need for Closure (NFC) questionnaire, and to identify which of two climate-change related goals their concepts addressed. They were specifically asked to clarify how each of their concepts can be used to either decrease the rate of climate change, or cope with the results of climate change.

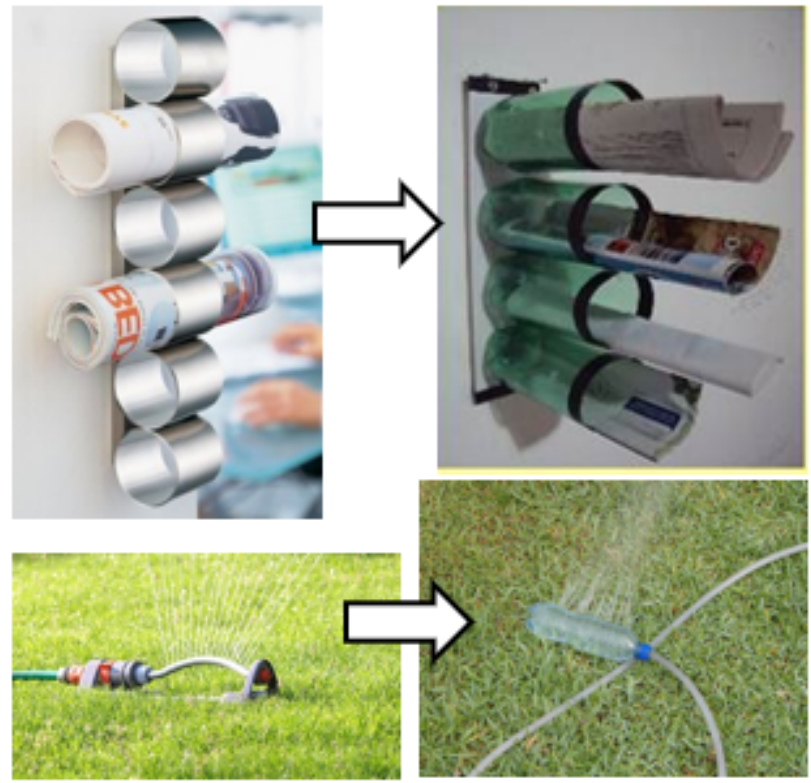

Figure 3. SCAMPER plastic-bottle reuse examples for Adapt (Top left image used with permission of APlaceForEverything.co.uk; Bottom right image used under https://creativecommons.org/licenses/by-nc-nd/2.0/).

To reduce instances of inaccurate claims, participants were reminded that reusing the material alone, e.g., as art, count as neither preventing nor coping with climate change.

\section{B. Results}

Table 1 compares by condition the average number of concepts generated per participant (including those who did not generate any concepts), as well as per concept-generating participant. The SCAMPER intervention led to fewer concepts compared to the control condition for both groups of participants, but this difference is significant for neither.

TABle 1. Average Number of Concepts Per Participant

\begin{tabular}{|c|c|c|c|}
\hline & SCAMPER & Control & $\mathbf{p}$ \\
\hline $\begin{array}{c}\text { Concept-generating } \\
\text { participants }\end{array}$ & 2.54 & 2.95 & 0.12 \\
\hline All participants & 1.14 & 1.64 & 0.10 \\
\hline
\end{tabular}

Fig. 4 compares between conditions, the percentage of participants who generated concepts for none, to all four, of the four wind-turbine-blade parts. The first pair of bar graphs correspond to the number of participants who did not generate any concepts: 16/36 (44.4\%) control and 16/29 (55.2\%) SCAMPER participants. Combining the latter four bar graphs for the SCAMPER condition, 13 of 29 participants (44.8\%) were able to generate reuse concepts for one or more of the four windturbine parts. In contrast, 20 of $36(55.6 \%)$ control-condition participants generated concepts for one or more of the four parts. The $10.7 \%$ difference between conditions in the proportion of participants who were able generate concepts for at least one part is insignificant $(\mathrm{p}=0.38)$. Thus, the SCAMPER condition was not successful in increasing the proportion of participants who were able to generate at least one reuse concept, and in fact decreased the proportion. 


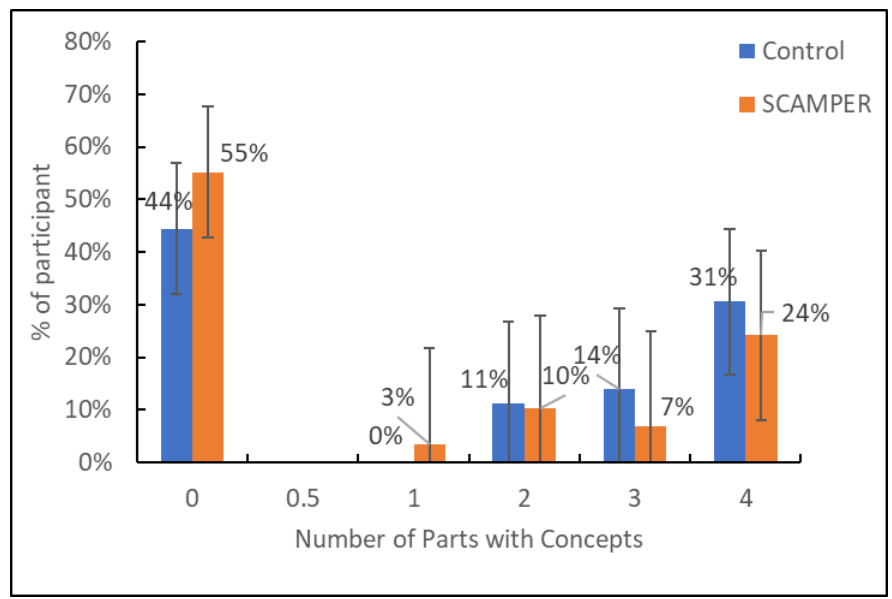

Figure 4. Proportion of participants who generated concepts for 0 to 4 of the four wind-turbine blade parts.

After removing the participants who produced zero concepts, Fig. 5 compares the proportion of participants who produced concepts for just one, up to all four of the parts. On average, each concept-generating participant in the control condition developed concepts for $3.35 / 4$ parts, while SCAMPERcondition participants generated concepts for $3.15 / 4$ parts, which is an insignificant difference.

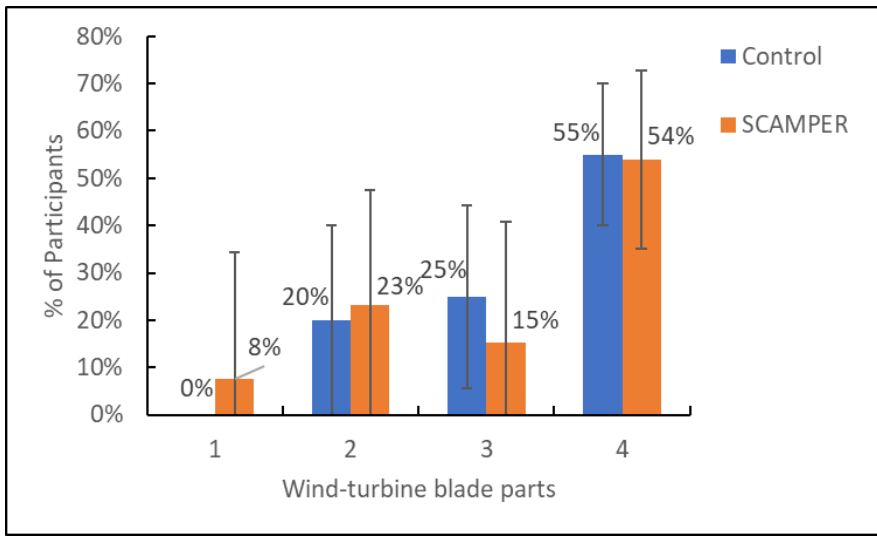

Figure 5. Proportion of concept-producing participants who generated concepts for just 1 up to all 4 of the four wind-turbine blade parts.

Fig. 6 compares the proportion of concepts generated for Parts 1 through 4 of the wind-turbine blade, which were always presented in the same order. As expected, more concepts are generally produced for the first versus last part presented, likely due to participant fatigue.

Table 1 compared by condition the average number of unique concepts generated per participant. However, participants often identified similar reuses for different windturbine parts, e.g., used both Parts 2 and 3 as fences. For concepts repeated by a participant, the one count for that same concept was allocated between the parts where the concept appears.

The SCAMPER method increased neither the number of concepts generated to reuse the wind-turbine blade parts, nor the proportion of participants who were able to generate concepts. However, the reuse concepts generated both with and without SCAMPER were categorized towards another possible method to support concept generation, as described in the next section.

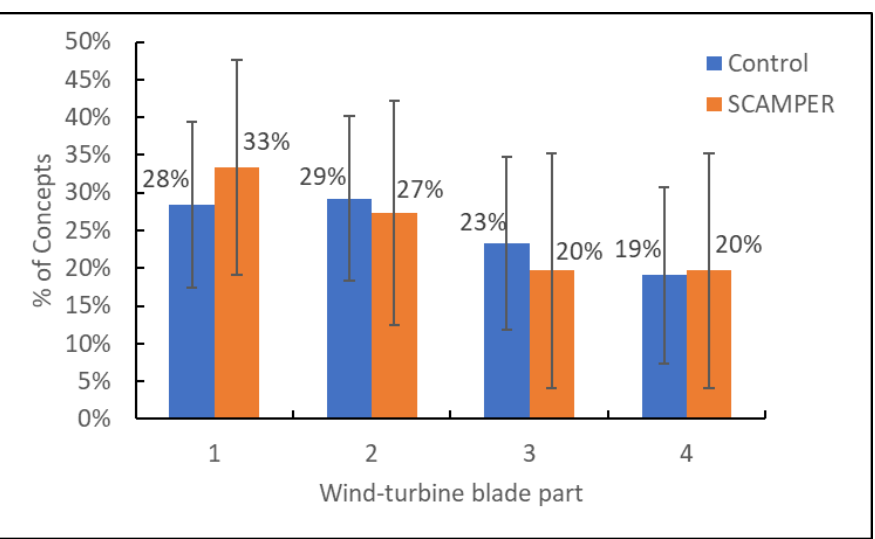

Figure 6. Proportion of concepts generated for wind-turbine-blade Parts 1-4.

\section{Iteration Five Using Perspective TAKing}

Building on previous iterations, a fifth iteration was conducted at Technische Universität Braunschweig in Germany.

\section{A. Methods}

Several approaches to categorizing the viable concepts from previous iterations were considered. One approach that emerged as promising for not just categorization, but also concept generation, involved perspective taking. This approach was then developed and tested in the current iteration on whether it can support participants to develop reuses for wind-turbine blades.

Participant responses in the fourth iteration also suggested a language barrier as non-English notes were found on the worksheets. To reduce this possible language barrier in the fifth iteration, the worksheets were translated to German.

\section{1) Participants}

Two hundred and four (204) participants were recruited from a second-year machine-elements course in mechanical engineering at TU Braunschweig. Demographic information, e.g., gender, native language and program of study, were collected along with their concepts and NFC responses. Of the participants who generated concepts, $85 \%$ identified as male, $10 \%$ identified as female, and $5 \%$ did not select from these choices. With respect to language proficiency, $87 \%$ were native speakers of German. Since all participants had attended a German university for at least one year, they were assumed to be able to understand and follow all the instructions in German.

\section{2) Intervention: Perspective-Taking Method}

In a way, the reorientation cue developed by Olteteanu and Shu (2018) for everyday objects was modified to support participants in developing reuses for much-larger wind-turbine parts. Rather than ask participants to reorient wind-turbine blade parts, they were asked to imagine the part on six different sides of them (above, below, front, back, left, right). In other words, participants were asked to mentally place the parts around themselves and view the parts from different perspectives. The perspective-taking intervention will also be referred to as the perspective method or condition below.

Fig. 7 shows how the perspective-taking intervention was conveyed graphically to participants, using "Teil", the German 
word for part, in a single-page aid. Compared to the four-page aid for SCAMPER in the fourth iteration, the perspective aid sheet is concise and requires minimal reading. The figures shown in Fig. 7 were revised multiple times to maximize neutrality and reduce suggestion of specific concepts.

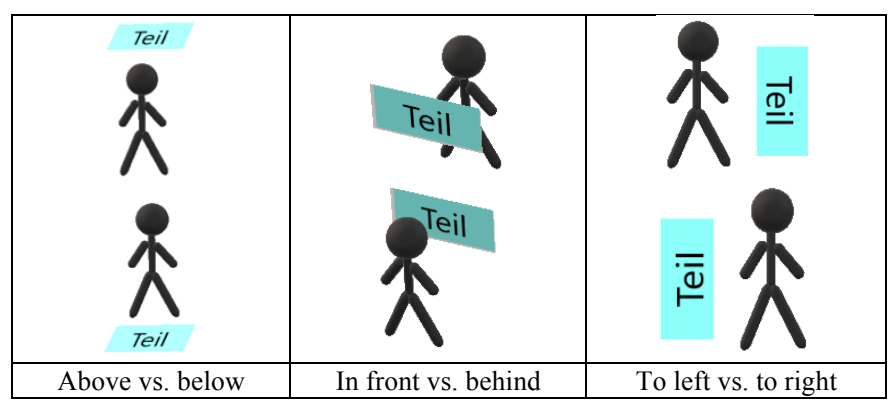

Figure 7. How the perspective-taking intervention was conveyed.

\section{3) Instructions to Participants}

The importance of wind-turbine-blade reuse was first conveyed through a presentation that highlighted the expected amount of waste generated from retired wind-turbine blades. Participants were then provided with worksheets that showed views of the four wind-turbine parts, with one part on each page. As in the two previous iterations, the worksheets asked participants to choose between and explain how their concepts either allow people to reduce the rate of climate change, or protect people from the effects of climate change.

Participants were asked to represent their concepts by simple sketches and minimize required cutting to the parts. They were also reminded that reusing the material alone counts towards neither preventing nor coping with climate change.

\section{4) Distribution of Worksheets}

Of the 204 participants, 98 were given the perspectivevisualization method shown in Fig. 7 as the intervention, or perspective condition. The remaining 106 were not given the intervention and comprise the control group or condition. Worksheets with the two conditions were alternatively distributed to participants. That is, participants should have received a condition that is different from their immediate neighbors. Finally, worksheets were collected at the end of the 45-minute limit given for generating and explaining concepts.

\section{B. Results}

To evaluate participants' concepts, their responses were first translated to English. The generated reuse concepts were then analyzed and classified. For concepts repeated by the same participant, the count for that same concept is allocated between the parts for which the concept appears, as demonstrated in Table 2. Table 3 compares by condition, the average number of unique concepts generated per participant by all participants (including those who did not generate any concepts), versus only the participants who generated at least one concept.
TABLE 2: EXAMPLE CONCEPT ALLOCATION TO WIND-TURBINE-BLADE PARTS

\begin{tabular}{|c|c|c|}
\hline Part & Concepts & Points \\
\hline Part 1 & Roof for a house & 1 \\
\hline Part 2 & Wind barrier, ship's hull & 1.5 \\
\hline Part 3 & Wind barrier & 0.5 \\
\hline Part 4 & Dike reinforcement & 1 \\
\hline
\end{tabular}

TABle 3: Average Number of Concepts Generated Per Participant

\begin{tabular}{|l|c|c|c|}
\hline & Perspective & Control & p \\
\hline $\begin{array}{l}\text { Concept generating } \\
\text { participants }\end{array}$ & 2.81 & 3.10 & 0.29 \\
\hline All participants & 1.91 & 1.55 & 0.043 \\
\hline
\end{tabular}

Considering only the concept-generating participants, the perspective group produced fewer concepts compared to the control condition, although this difference is insignificant. This non-intuitive trend could be because participants who persisted (instead of abandoned) the control condition were intrinsically stronger designers, and thus able to generate more concepts.

What was significant, is that the perspective group produced more concepts compared to the control condition when averaged over all participants. That is, when including all participants, the perspective group has a 0.36 higher average number of concepts than the control group. This difference is significant with a 1tailed t-test at $\mathrm{p}=0.043$, supporting the hypothesis that perspective-taking helps participants to generate concepts.

Furthermore, in the perspective condition, 72 of 106 participants $(67.9 \%)$ were able to generate reuse concepts for one or more of the four wind-turbine parts. In contrast, 49 of 98 $(50.0 \%)$ control-condition participants generated concepts for one or more of the four parts. The $17.9 \%$ difference between the proportion of the two groups who were able generate concepts for at least one part is significant ( $\mathrm{p}=0.0095,95 \%$ Confidence Interval: $4.40 \%$ to $30.6 \%$ ). Thus, the perspective method was successful in increasing the proportion of engineering-student participants who were able to generate at least one reuse concept.

Fig. 8 compares by condition, the proportion of participants who generated concepts for zero up to four of the wind-turbineblade parts. Consistent with the above results, a higher number of participants in the control condition returned worksheets with no concepts. However, when analyzing participants who did generate at least one concept, the gap between conditions reduces. Fig. 9 compares by condition the number of windturbine parts for which concept-generating participants identified reuses. On average, each concept-generating participant in the control condition identified reuses for 2.80/4 parts while perspective-condition participants identified reuses for $2.72 / 4$ parts, an insignificant difference.

The difference between the average number of parts with concepts and the average number of unique concepts (Table 3) is due to a combination of repetition and multiple concepts per part. Participants would generate similar concepts for different wind-turbine parts, or multiple concepts per part. 


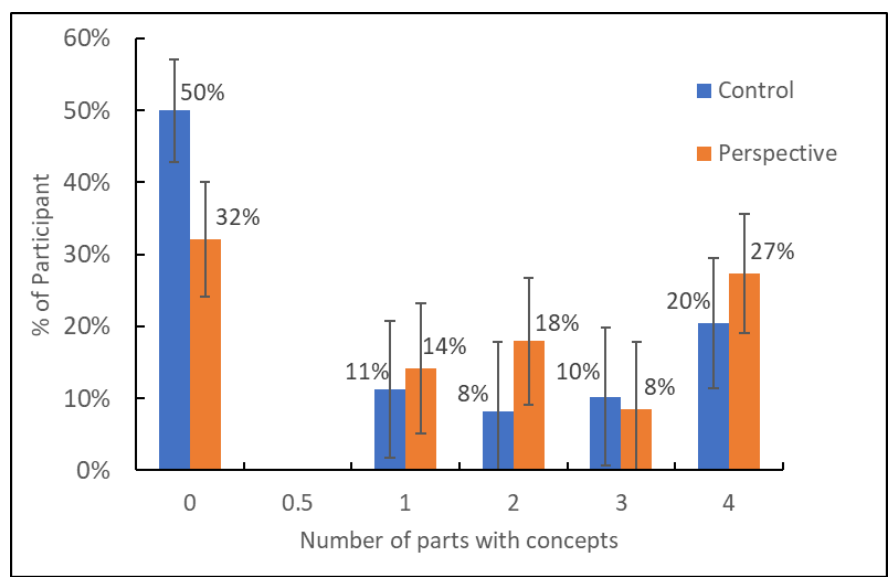

Figure 8. Proportion of participants who generated concepts for 0 to 4 of the four wind-turbine blade parts.

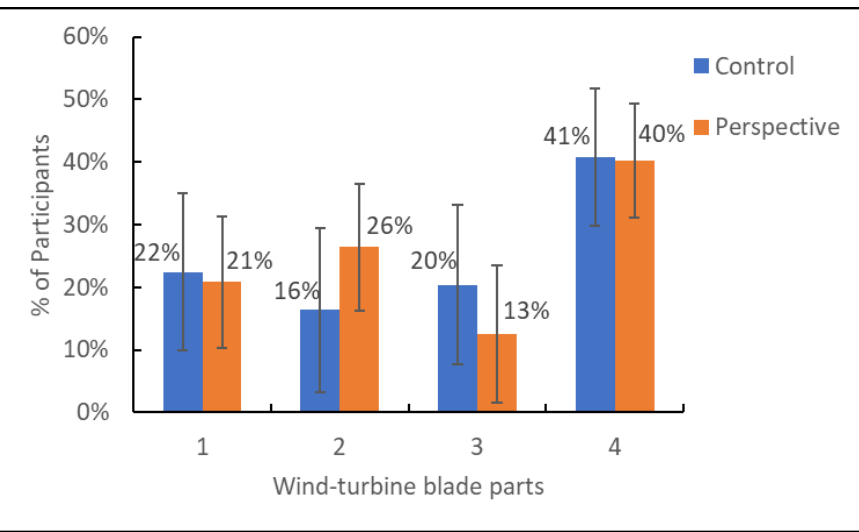

Figure 9. Proportion of concept-producing participants who generated concepts for just 1 to all 4 of the four wind-turbine-blade parts.

Similar to the previous (fourth) iteration, since participants went through worksheet pages ordered corresponding to windturbine-blade Parts 1 to 4 , the number of concepts generated for each subsequent part decreased as shown in Fig. 10. This result is likely due to participant fatigue. Again, for concepts repeated by the same participant, the count for that same concept is allocated between the parts where the concept appears.

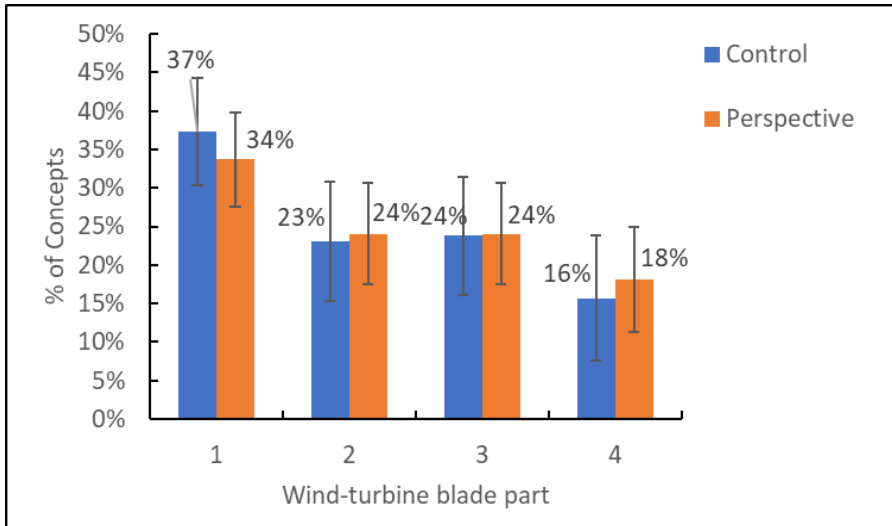

Figure 10. Proportion of concepts generated for wind-turbine-blade Parts 1-4.

\section{DISCUSSION}

\section{A. SCAMPER vs. Perspective-Taking Conditions}

Comparing the SCAMPER group of the fourth iteration and the perspective-taking group of the fifth iteration yields a $23 \%$ difference in proportion of participants who produced no concepts ( $\mathrm{p}=0.024,95 \%$ Confidence Interval: $3.05 \%$ to $41.3 \%$ ). This significant difference suggests the benefit of the perspective-taking method by comparing it against an existing method as an intervention. Furthermore, the corresponding proportions for the control condition in the fourth iteration that used SCAMPER was $44 \%$, versus $50 \%$ for the control condition in the fifth iteration that used the perspective-taking method. The difference of only $6 \%$ in the control-group participants between iterations of the study that used different interventions is insignificant $(\mathrm{p}=0.54)$. Fig. 11 shows the proportion of participants who produced no concepts for both conditions of the fourth and fifth iterations of the study.

Limitations of this comparison include the following. While both the SCAMPER and perspective-taking iterations were performed at TU Braunschweig's mechanical engineering department, participants were from different classes and years of study. Moreover, the SCAMPER materials were provided in English vs. German for the perspective-taking materials. Finally, the length of the 4-page SCAMPER aid sheets was considerably longer than the 1-page perspective-taking aid sheet.

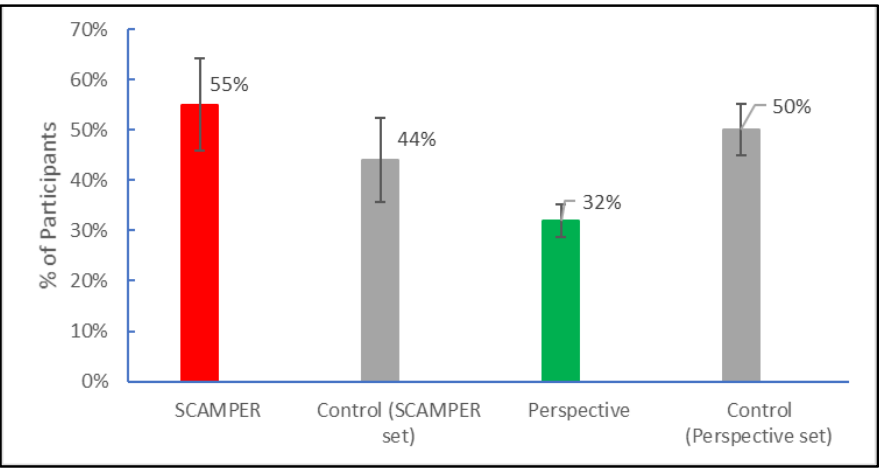

Figure 11. Proportion of participants who produced no concepts in fourth iteration using SCAMPER and fifth iteration using perspective-taking.

\section{B. Regulatory Focus Theory and Need for Closure}

As previously mentioned, participants were asked to develop concepts that either 1) Allow people to reduce the rate of climate change, or 2) Protect people from the effects of climate change.

The first choice corresponds to the promotion focus of Regulatory Focus Theory (RFT) by approaching hopes and gains, e.g., improvement of the environment. The second choice corresponds to the prevention focus of RFT, which is about preventing losses, e.g., protecting people.

Common promotion-focused concepts included: turbines for hydroelectric power stations; artificial coral reefs; and solar panel mounts. Concepts generated under the prevention-focus (protect people) category included: flood and wind barriers; dike reinforcement or erosion protection; sun covering; and emergency shelters.

In the perspective condition of the fifth iteration, 65 (of 106) participants completed the Need for Closure (NFC) 
questionnaire. Linear regression yielded a negative relationship between the ambiguity subscale of NFC and the proportion of promotion-focused concepts. That is, the higher a participant's desire to avoid ambiguity, the fewer promotion-focused concepts they produced as a proportion of all the concepts they generated. Shown in Table 4, a medium negative correlation ( $\mathrm{r}$ $=-0.31$ ) was found between participants' ambiguity subscale and their tendency to produce promotion-based concepts. This finding is related to the work of Toh and Miller (2014), who found that creativity is affected by varying tolerance levels for ambiguity.

TABLE 4: REGRESSION RESULTS FOR PERSPECTIVE-CONDITION PARTICIPANTS' NFC AMBIGUITY SUBSCALE AND PROPORTION OF PROMOTION-FOCUSED CONCEPTS

\begin{tabular}{|c|c|c|c|}
\hline Coefficients & Estimate & $\begin{array}{c}\mathbf{9 5 \%} \text { Confidence } \\
\text { Interval }\end{array}$ & $\mathbf{p}$ \\
\hline Intercept & 1.38 & 0.633 to 2.13 & 0.0005 \\
\hline Ambiguity & -0.0237 & -0.0431 to -0.00424 & 0.018 \\
\hline
\end{tabular}

$\mathrm{F}=5.96, \mathrm{R}^{2}=0.0961, \mathrm{r}=-0.31$

This same trend was not seen in the control condition of the fifth iteration. Instead, the proportion of promotion-based concepts correlated with the decisiveness subscale of the NFC. That is, the more decisive the participant, the more promotionfocused concepts they generated. Analysis of the 46 participants with complete NFC scores yielded a medium positive correlation between their decisiveness subscale and the proportion of promotion-based concepts they generated, as shown in Table 5.

TABLE 5: REGRESSION RESULTS FOR CONTROL-CONDITION PARTICIPANTS' NFC DECISIVENESS SUBSCALE AND PROPORTION OF PROMOTION-FOCUSED CONCEPTS

\begin{tabular}{|c|c|c|c|}
\hline Coefficients & Estimate & $\begin{array}{c}\mathbf{9 5 \%} \text { Confidence } \\
\text { Interval }\end{array}$ & $\mathbf{p}$ \\
\hline Intercept & -0.336 & -1.133 to 0.463 & 0.40 \\
\hline Decisiveness & 0.0284 & -0.0004 to -0.0572 & 0.053 \\
\hline
\end{tabular}

$\mathrm{F}=4.84, \mathrm{R}^{2}=0.131, \mathrm{r}=0.36$

Overall, only 75 of 204 (48 perspective- and 27 controlcondition) participants explicitly identified whether their concepts aimed to achieve promotion-focused versus prevention-focused goals. Some uncategorized concepts were analyzed and added to the two categories. Overall, the control group generated 42 prevention-based concepts and 25 promotion-based concepts, and the perspective group generated 65 prevention-based concepts and 67 promotion-based concepts. Therefore, the perspective condition appeared to help balance the categories.

\section{Effects of Conditions on Shredding Concepts}

While participants were discouraged from making excessive cuts to wind-turbine blade parts, some participants suggested incineration or shredding the parts for use as insulation.

In the fifth iteration, $2 \%$ of the perspective-taking participants generated concepts which required shredding compared to $7 \%$ in the control condition. This $5 \%$ decrease $(\mathrm{p}=$
0.011) suggests that the perspective-taking method encouraged reuses that utilize the overall shape of the part, by asking participants to imagine the parts around them.

In the fourth iteration using the SCAMPER intervention, too few (3) concepts that involve shredding were observed over both conditions combined; therefore, no conclusions can be drawn.

\section{Prevention-Based vs. Promotion-Based Example Reuses}

Using wind-turbine-blade parts in shelter construction is a common participant-generated concept. Fig. 12 shows a busshelter concept generated by a participant in the perspectivetaking group. The participant selected the box indicating that this concept is for protecting people from climate change, i.e., the prevention-focus choice. In the perspective-taking condition, 27 of $76(35.5 \%)$ concept-generating participants produced ideas involving shelter or roofing. In the control condition, 16 of 49 $(32.6 \%)$ participants produced similar concepts.

Fig. 13 presents a unique (control-condition) participant's concept of a vertical planter for plants to absorb carbon dioxide. The participant selected the box indicating that this concept is for enabling people to reduce the rate of climate change, i.e., the promotion-focus choice.

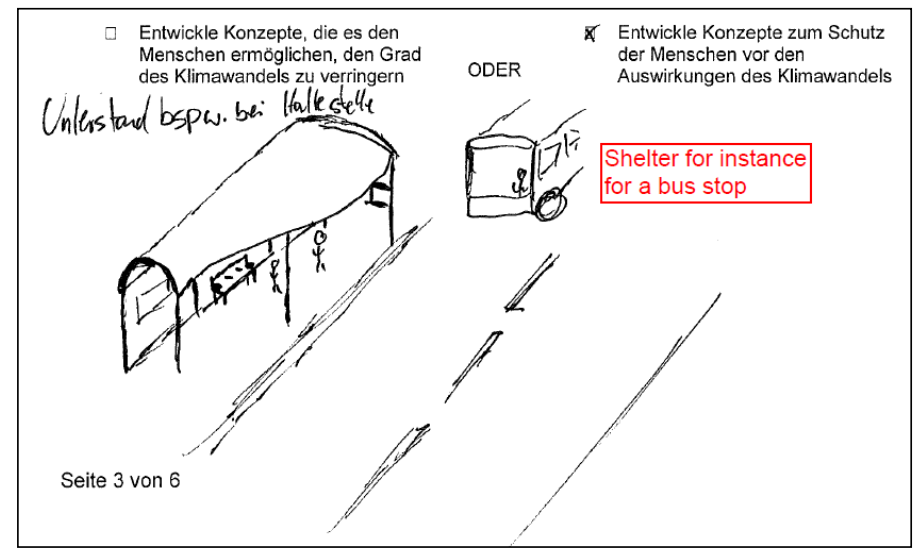

Figure 12. Participant-generated bus shelter concept for Part 1.

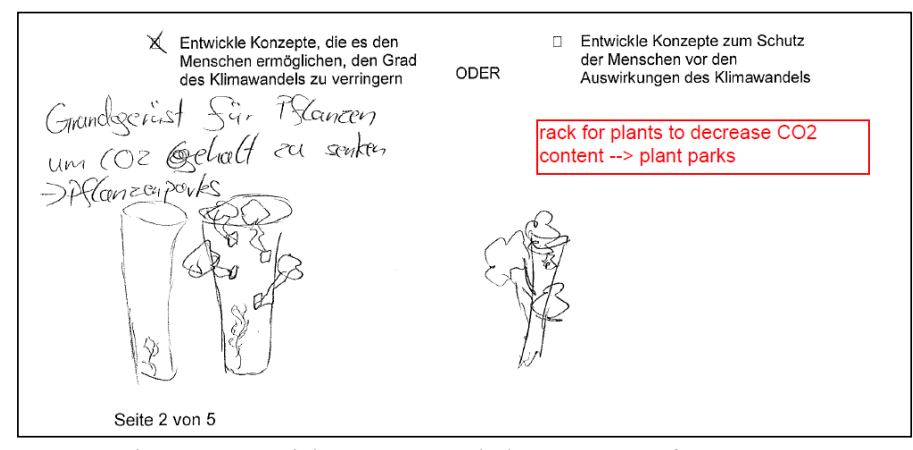

Figure 13. Participant-generated planter concept for Part 1.

\section{SUMMARY AND FUTURE WORK}

Five iterations of a study asked engineering-student participants to generate reuse concepts for wind-turbine blades. With focus on the fourth and fifth iterations, this paper examined the effect of different methods to support this challenging concept-generation activity. 
To reduce functional fixedness, the first iteration did not reveal that the parts shown were wind-turbine blades, nor were participants specifically prohibited from identifying reuses as wind-turbine blades. However, many participants identified reuses as wind-turbine blades or airplane parts, which are excluded for safety reasons. Thus, the second iteration revealed that the parts were wind-turbine blades, and prohibited their reuse in the same or airplane applications. While this restriction reduced these specific reuse applications, the resulting reuse concepts either already exist or were impractical. Kwon et al. (2018) report in more detail the first two iterations.

To help guide possible concepts, third-iteration participants were asked to develop concepts to address climate change. While this additional constraint provided direction and focus for concept generation, participants were observed to struggle to develop solutions. Thus, subsequent iterations explored methods to support this concept-generation activity.

The fourth iteration used SCAMPER, an existing method, to support engineering-student designers in concept generation. However, SCAMPER did not appear to aid in reuse-concept generation. Thus, the perspective-taking method was developed for the fifth iteration. This method was observed to reduce the number of participants who produced no concepts, but did not affect the number of parts for which concepts were generated, nor the number of concepts generated by participants who persisted in the study. However, participants were not specifically encouraged to maximize the number of concepts they generated.

While participants were asked to generate reuse concepts to address climate change, they were not given guidance to increase the environmental benefits of their concepts. Therefore, future work could also incorporate aspects to guide concepts towards those that maximize environmental benefits. Metrics to evaluate how well reuse concepts contribute to sustainability include manufacturing cost, waste management, and energy consumption (Jawahir \& Dillon, 2007).

This work aims to gain insights on the process of finding potential alternative uses of wind-turbine blades, which currently lack sustainable end-of-life solutions. The lack of sustainable post-use opportunities is recognized as a major impact area in sustainable manufacturing (Haapala et al., 2013). While attention to recycling and remanufacturing contributes to environmentally conscious product design, end-of-life options for already-designed and installed wind-turbine blades are required. Typical product end-of-life options include: direct reuse, repair, remanufacturing, recycling, and disposal (Ramani et al., 2010; Ilgin \& Gupta, 2010), with Sabbaghi \& Behdad (2018) noting that repair decisions are highly price dependent. Missing from these options is the opportunity to design for alternative uses of products at end-of-life. The role of product design in sustainable manufacturing has long focused on designing for the environment (Allen et al., 2002). In the case of closing the loop for wind-turbine blades, product design methods could also be leveraged towards alternative reuses of these parts, in the absence of other viable end-of-life solutions.

\section{ACKNOWLEDGMENTS}

The authors thank: Drs. Alexandra Pehlken (Oldenburg Univ.) and KlausDieter Thoben (Bremen Univ.); Elisa Kwon (Univ. of Toronto); Study participants and colleagues at TU Braunschweig; Prof. Gernot von Collani (Univ. of Leipzig) for his German translation of NFC questionnaires.

\section{REFERENCES}

Adamson R (1952) Functional Fixedness as Related to Problem Solving: A Repetition of Three Experiments. J Experimental psychology 44/4:288.

Allen D, Bauer D, Bras B, Gutowski T, Murphy C, Piwonka T, Sheng P, Sutherland J, Thurston D, Wolff E (2002) Environmentally benign manufacturing: Trends in Europe, Japan, and the USA. ASME J Manuf. Sci. Eng. 123/3:908-920.

Dinar M, Shah J, Cagan J, Leifer L, Linsey J, Smith S, Hernandez N (2015) Empirical studies of designer thinking: past, present, and future. J Mechanical Design, 137/2:021101.

Global Wind Energy Council (2016) Global Wind Report Annual Market Update. Brussels: Global Wind Energy Council.

Hauschild M (2017) personal communication. 22 August 2017. CIRP GA.

Haapala K, Zhao F, Camelio J, Sutherland J, Skerlos S, Dornfeld D, Jawahir I, Clarens A, Rickli J (2013) A review of engineering research in sustainable manufacturing. ASME J Manuf. Sci. Eng. 135/4: 041013.

Higgins E (2000) Making a Good Decision: Value from Fit. American Psychologist. 55/11:1217-1230.

Ilgin MA, Gupta SM (2010) Environmentally conscious manufacturing and product recovery (ECMPRO): A review of the state of the art. J Environmental Management 91: 563-591.

Jansson D, Smith S (1991) Design fixation. Design studies. 12/1:3-11.

Jawahir I, Dillon O (2007) Sustainable Manufacturing Processes: New Challenges for Developing Predictive Models and Optimization Techniques. Proc. First International Conference on Sustainable Manufacturing. 1-19.

Kudrowitz B, Dippo C (2013) When Does a Paper Clip Become a Sundial? Exploring the Progression of Novelty in the Alternative Uses Test. J Integrated Design \& Process Science. 17/4:3-18.

Kwon E, Pehlken A, Thoben KD, Bazylak A, Shu LH (2018) Visual similarity to aid alternative-use concept generation for retired wind-turbine blades, Proc. ASME IDETC, 26-29 Aug, Quebec City, Canada DETC2018-85670.

Lai S, Shu L (2016) Individual Differences in Tendency for Design Fixation, Design Computing \& Cognition, June 27-29, 321-338. Evanston, IL, USA.

Moreno D, Blessing L, Yang M, Hernández A, Wood K (2016) Overcoming design fixation: Design by analogy studies and nonintuitive findings. Artificial Intelligence for Eng. Design, Analysis \& Manufg. 30/2:185-199.

Olteteanu A, Shu L (2018) Object Reorientation and Creative Performance. J Mechanical Design. 140/3:031102-031102-9.

Ramani K, Ramanujan D, Bernstein W, Zhao F, Sutherland J, Handwerker C, Choi J, Kim H, Thurston D (2010) Integrated sustainable life cycle design: a review. J Mechanical Design. 132/9:1-15.

Ramanujan D, Bernstein W, Chandrasegaran S, Ramani K (2017) Visual analytic tools for sustainable lifecycle design: current strategies, challenges, and future opportunities. J Mechanical Design. 139/11:1-19

Sabbaghi M, Behdad S (2018) Consumer decisions to repair mobile phones and manufacturer pricing policies: The concept of value leakage. Resources, conservation, and recycling. 133/101-111

Ramirez-Tejeda K, Turcotte D, Pike S (2017) Unsustainable Wind Turbine Blade Disposal Practices in the United States: A Case for Policy Intervention and Technological Innovation. NEW SOLUTIONS: J Environmental and Occupational Health Policy. 26/4:581-598.

Toh C, Miller S (2014) The role of individual risk attitudes on the selection of creative concepts in engineering design. ASME DETC2014-35106.

Viswanathan V, Tomko M, Linsey J (2016) A study on the effects of example familiarity and modality on design fixation. AI EDAM. 30/2:171-184.

Wang J, GangaRao H, Liang R, Liu W (2015) Durability and prediction models of fiber-reinforced polymer composites under various environmental conditions: A critical review. J Reinf. Plastics \& Composites. 35/3:179-211.

Webster D, Kruglanski A (1994) Individual Differences in Need for Cognitive Closure. J Personality and Social Psychology. 67/6:1049-1062. 